\title{
Micropropagation of Echinacea angustifolia, $E$. pallida, and $E$. purpurea from Stem and Seed Explants
}

\author{
James F. Harbage ${ }^{1}$ \\ Horticulture, Forestry, Landscape, and Parks Department, South Dakota \\ State University, Box 2140A, Brookings, SD 57007-0996 \\ Additional index words. purple coneflower, tissue culture, contamination, seed dormancy, \\ shoot multiplication, rooting
}

\begin{abstract}
Micropropagation of three Echinacea species, E. angustifolia DC., E. pallida Nutt., and $E$. purpurea Moench., was investigated as a potential means of germplasm preservation of species faced with overcollection in the wild and rapid clonal propagation of elite individuals with unique medicinal or ornamental properties. Very high contamination rates occurred with shoot-tip explants but not with nodal segments. Contamination rates for seed explants were inversely related to the number of seedcoat layers removed, ranging from $100 \%$ contamination from intact seeds to near $0 \%$ contamination from excised embryos. Dormancy of seed explants was also eliminated when the pericarp and integument were removed. Addition of benzyladenine (BA) to the culture medium induced shoot multiplication and inhibited root formation in all three species. Shoot multiplication rates were low (1-3 shoots per culture) when seed explants were placed on a medium with BA levels low enough to avoid adventitious shoot formation $(0.45 \mu \mathrm{M})$. Shoot count was higher on half-strength Murashige and Skoog (MS) minerals, while leaf size was greater on full-strength MS minerals. Cultures did not perform well in Woody Plant Medium. Reducing subculture frequency from 4 to 2 weeks increased shoot multiplication from 1.4 to 1.8 shoots per subculture and total shoots produced per subculture after 12 weeks from 2.8 to 23.9. Rooting occurred readily on shoots isolated from $E$. purpurea cultures and was not promoted by addition of IBA to the rooting medium. Rooting was low and nil on shoots from cultures of $E$. angustifolia and $E$. pallida, respectively. Methods described in this study allow rapid multiplication of three Echinacea species and subsequent rooting of $E$. purpurea. Future improvements in root induction treatments will allow these methods to be used effectively for micropropagation and maintenance of disease-free germplasm of Echinacea species. Chemical names used: $N$-(phenylmethyl)-1H-purine-6-amine (BA); $1 \mathrm{H}$-indole-3-butyric acid (IBA).
\end{abstract}

Echinacea, purple coneflower, is a genus of herbaceous perennials, including nine species primarily indigenous to the Central and Plains States (McGregor, 1968). It has received considerable attention in recent years for its ornamental value in the landscape and in florist bouquets and for its medicinal qualities. This popularity has led to a need for improvements in propagation methods over traditional seed propagation and crown division to facilitate faster multiplication and support rapid availability of elite clonal selections.

Several Echinacea species have high economic value. Echinacea purpurea has long

\footnotetext{
Received for publication 28 June 1999. Accepted for publication 21 Feb. 2000. South Dakota Agricultural Experiment Station publication 3138. Research funded from the South Dakota Agricultural Experiment Station project no. SD00403-H. Mention of trade names does not imply endorsement of the products named nor criticism of similar ones not named. The cost of publishing this paper was defrayed in part by the payment of page charges. Under postal regulations, this paper therefore must be hereby marked advertisement solely to indicate this fact. ${ }^{1}$ Current address: Longwood Gardens, P.O. Box 501, Kennett Square, PA 19348. E-mail address: jharbage@longwoodgardens.org
}

E. angustifolia has higher value per kilogram of root.

High value of Echinacea has led to species endangerment problems because of harvesting of native colonies for sale to pharmaceutical companies (Foster, 1990). Two of the nine species (E. tennesseensis Small. and E. laevigata Blake.) are on the federal endangered species list or are being considered for this list. Efforts are currently under way to multiply these species for reestablishment in the wild. Ornamental and medicinal value of the midwestern species, E. angustifolia, E. pallida, and E. purpurea, may also lead to their endangerment through overharvesting of native populations.

Most Echinacea species are easily seedpropagated following stratification. Seedpropagated plants often will not flower until the second year. Many years are required for the number of generations of self-pollination required to achieve inbred lines, and some species, such as E. angustifolia, are self-incompatible. Inherent heterozygosity and tendency toward interspecific hybridization often require vegetative propagation to maintain superior characteristics of select individuals. Echinacea angustifolia and E. pallida produce thick taproots that can be propagated as root cuttings, while $E$. purpurea has a fibrous root system and is best vegetatively propagated by crown division or basal stem cuttings. Micropropagation offers improvements over traditional vegetative propagation because of the faster rate of multiplication (Lineberger, 1983), and can also be effective in propagating species that are troublesome to clone by conventional means (Bridgen, 1986). This study was undertaken to investigate micropropagation for rapid multiplication of Echinacea species.

been a popular garden ornamental and was rated one of the top-selling perennials surveyed from 1992 through 1995 (Rhodus, 1995). The cultivar Magnus was voted the perennial plant of the year in 1998 by the Perennial Plant Association. Echinacea purpurea has been a prolific producer of high-quality, longstemmed flowers and was ranked third of 19 species for potential profitability as a fieldgrown florist crop (Starman et al., 1995). In addition to ornamental value, Echinacea is one of the most important medicinal herbs in commerce today (Foster, 1985), and has a long history of medicinal use dating back to the Plains Indian tribes of midwestern America (Foster, 1990; Kindscher, 1989; Shemluck, 1982). Medicinal effectiveness apparently stems from the presence of several endogenous compounds effective in immuno-stimulation (Kindscher, 1989). Use of medicinal Echinacea products is at an all-time high in Europe and North America (Rawls, 1996). Echinacea-based products are the bestselling herbal remedies in the United States, with $9.1 \%$ of the market share. Commercial production of Echinacea for medicinal products in the United States and Canada involves $E$. purpurea (80\% of planted area) and $E$. angustifolia (20\% of planted area). However,

\section{Materials and Methods}

Experiment 1. Isolation of aseptic cultures from seedling and stem explants. Aseptic explant isolation was compared with seed and stem explants. Seeds of $E$. angustifolia, $E$. pallida, and E. purpurea 'Bravado' were isolated using four methods: 1) intact; 2) after removing the outer seedcoat (pericarp); 3) after removing the pericarp plus the integument layer; and 4) after removing the pericarp plus the integument layer plus the endosperm layer (excised embryos). One-centimeter shoot tip and stem explants were also evaluated. These were collected prior to flower development from actively growing, 2-year-old $E$. purpurea 'Bravado' and E. angustifolia stems that were 15 to $30 \mathrm{~cm}$ long. Stock plants were grown in a greenhouse in pasteurized 2 polystyrene : 2 sphagnum peat moss : 1 clay-loam field soil media in 15-cm-diameter, standard, round plastic pots. Plants were watered as needed and fertilized weekly with $300 \mathrm{mg} \cdot \mathrm{L}^{-}$ ${ }^{1} 20 \mathrm{~N}-8.7 \mathrm{P}-16.6 \mathrm{~K}$ soluble Peters brand fertilizer (Grace Sierra Horticultural Products Co., Allentown, Pa.). All explants were treated with a $15 \%$ household bleach solution (final concentration was $0.8 \%$ sodium hypochlorite) containing $0.1 \%$ Tween $20^{\circledR}$ detergent; they 
were submerged in the solution with agitation for $15 \mathrm{~min}$ followed by rinsing for $30 \mathrm{~s}$ in sterile deionized water. Explants were then placed aseptically on sterile medium containing Murashige and Skoog (MS) (1962) minerals, $0.56 \mathrm{~mm}$ myoinositol, $1.20 \mu \mathrm{m}$ thiamine $\mathrm{HCl}, 4.44 \mu \mathrm{M}$ BA, $87.6 \mathrm{~mm}$ sucrose, and $0.7 \%$ Bacto agar(Difco Laboratories, Detroit). These ingredients were used in all experiments except where otherwise stated. Cultures were incubated in $40 \mu \mathrm{mol} \cdot \mathrm{m}^{-2} \cdot \mathrm{s}^{-1}$ continuous coolwhite fluorescent light at $25^{\circ} \mathrm{C}$. Cultures were evaluated 4 weeks after initiation and data were taken on percent contamination and germination, and number of shoots produced (shoot count). A completely random design was used with four individual cultures (one explant per culture) representing one replicate and three replicates for each treatmentspecies combination. All data were analyzed by analysis of variance. Analysis of aseptic explant isolation and establishment data was performed on two subsets of the entire data set. The first analysis included only treatments involving seed explants and data on contamination, germination, and shoot count. The second analysis included treatments involving both seed and vegetative explants and data on contamination and shoot count.

Experiment 2. Cytokinin influence on shoot formation. Initially, shoot formation in response to a wide range of concentrations of BA was evaluated using excised embryos of $E$. angustifolia, E. pallida, and E. purpurea 'Bravado'. Embryos were disinfested and placed on media as described above but modified to include $0.45,4.45$, and $44.5 \mu \mathrm{M}$ BA. Cultures were evaluated after 4 weeks of incubation as described above for shoot count and percent adventitious shoots (shoots not arising from nodes). A randomized complete-block design was used.

Shoot formation in response to a lower cytokinin range was evaluated using the same procedure but with $0,0.09,0.9$, and $8.9 \mu \mathrm{MBA}$. A completely random design was used. Shoot number, leaf length, and root number were recorded after 4 weeks of culture.

Experiment 3. Mineral composition and concentration influence on shoot formation. Shoot formation in response to mineral composition and concentration was evaluated with excised embryos of E. angustifolia, E. pallida, and E. purpurea 'Bravado'. Explants were placed on the medium described above, in which mineral composition was modified to include MS minerals or Woody Plant Medium (WPM) (Lloyd and McCown, 1980) minerals at normal concentration or at one-half normal concentration. Cultures were arranged in a completely random design. Shoot number, leaf length, and root number were recorded after 4 weeks of culture.

Experiment 4. Shoot formation response to subculture frequency. Influence of subculture frequency on shoot formation was investigated with established (seed explants isolated and subcultured nine times at 4-week intervals) E. angustifolia shoot cultures. Established cultures were divided and single shoots placed on fresh medium using MS minerals

and BA at $0.9 \mu \mathrm{M}$. Cultures were subdivided and transferred to fresh medium at 2-, 3-, and 4-week intervals. Cultures were arranged in a completely random design. Data were recorded for shoots per subculture and total shoots produced after 12 weeks.

Experiment 5. Auxin, light, and temperature influence on rooting. Influence of auxin, light, and temperature on root formation from established shoot cultures (after more than 15 subcultures) of E. angustifolia, E. pallida, and E. purpurea 'Bravado' was investigated using a two-phase rooting system. Individual shoots were excised from proliferating shoot cultures and placed in sterile medium containing 43.8 mm sucrose, 1.95 mm MES [2( $N$-morpholino)

ethanesulfonic acid], $7 \mathrm{~g} \cdot \mathrm{L}^{-1}$ Difco Bactoagar, and $0,1.5$, and $15.0 \mu \mathrm{M}$ IBA. Media $\mathrm{pH}$ was adjusted to 5.5. Shoots were incubated at 21 or $30^{\circ} \mathrm{C}$ in the dark or under $40 \mu \mathrm{mol} \cdot \mathrm{m}^{-2} \cdot \mathrm{s}^{-}$ ${ }^{1}$ continuous cool-white fluorescent light. Data were taken on root count and percent shoots with roots.

\section{Results}

Experiment 1. Isolation of aseptic cultures from seedling and nodal explants. Contamination was affected significantly by an interaction between species and seed covering removal (Table 1). Contamination level dropped as the number of seed coverings removed

Table 1. Influence of seed covering removal and species on contamination, germination, and shoot count from newly isolated seed explants of three Echinacea species.

\begin{tabular}{|c|c|c|c|c|}
\hline $\begin{array}{l}\text { Seed covering } \\
\text { layers removed }\end{array}$ & Species & $\begin{array}{c}\text { Contamination } \\
(\%)\end{array}$ & $\begin{array}{c}\text { Germination } \\
(\%)\end{array}$ & $\begin{array}{c}\text { Shoots } \\
\text { per explant }\end{array}$ \\
\hline \multirow[t]{3}{*}{$\overline{0}$} & E. angustifolia & $72 \mathrm{a}^{2}$ & & \\
\hline & E. pallida & $11 \mathrm{~b}-\mathrm{d}$ & $50 \mathrm{n}$ & $0.5 \mathrm{n}$ \\
\hline & E. purpurea 'Bravado' & $39 \mathrm{~b}$ & & \\
\hline \multirow[t]{3}{*}{1} & E. angustifolia & $39 \mathrm{~b}$ & & \\
\hline & E. pallida & $33 \mathrm{bc}$ & $73 \mathrm{~m}$ & $0.8 \mathrm{mn}$ \\
\hline & E. purpurea 'Bravado' & $19 \mathrm{~b}-\mathrm{d}$ & & \\
\hline \multirow[t]{3}{*}{2} & E. angustifolia & $0 \mathrm{~d}$ & & \\
\hline & E. pallida & $0 \mathrm{~d}$ & 971 & $1.0 \mathrm{~m}$ \\
\hline & E. purpurea 'Bravado' & $8 \mathrm{~cd}$ & & \\
\hline \multirow[t]{3}{*}{3} & E. angustifolia & $0 \mathrm{~d}$ & & \\
\hline & E. pallida & $0 \mathrm{~d}$ & 1001 & 1.51 \\
\hline & E. purpurea 'Bravado' & $0 \mathrm{~d}$ & & \\
\hline \multicolumn{5}{|l|}{ ANOVA } \\
\hline Source of variation & df & & Mean square & \\
\hline$\overline{\text { Species (S) }}$ & 2 & $864.32^{*}$ & $198.79^{\mathrm{Ns}}$ & $0.06^{\mathrm{NS}}$ \\
\hline \multicolumn{5}{|l|}{ Seed covering } \\
\hline $\mathrm{S} \times \mathrm{SC}$ & 6 & $772.22^{*}$ & $437.99^{\mathrm{Ns}}$ & $0.18^{\mathrm{Ns}}$ \\
\hline Error & 24 & 235.38 & 335.86 & 0.11 \\
\hline
\end{tabular}

${ }^{2}$ Mean separation within columns by Duncan's multiple range test, $P \leq 0.05$.

Ns, *,****Nonsignificant or significant at $P \leq 0.05$ or 0.001 , respectively.

Table 2. Influence of explant source and species on contamination and shoot count from newly isolated cultures of two Echinacea species.

\begin{tabular}{|c|c|c|c|}
\hline Explant source & Species & $\begin{array}{c}\text { Contamination } \\
(\%)\end{array}$ & $\begin{array}{c}\text { Shoots } \\
\text { per explant }\end{array}$ \\
\hline Intact seed & $\begin{array}{l}\text { E. angustifolia } \\
\text { E. purpurea 'Bravado' }\end{array}$ & $\begin{array}{l}72 b^{2} \\
39 c\end{array}$ & $0.4 \mathrm{n}$ \\
\hline $\begin{array}{l}\text { Seed minus one } \\
\text { covering layer }\end{array}$ & $\begin{array}{l}\text { E. angustifolia } \\
\text { E. purpurea 'Bravado' }\end{array}$ & $\begin{array}{l}39 \mathrm{c} \\
19 \mathrm{~cd}\end{array}$ & $0.9 \mathrm{~m}$ \\
\hline $\begin{array}{l}\text { Seed minus two } \\
\text { covering layers }\end{array}$ & $\begin{array}{l}\text { E. angustifolia } \\
\text { E. purpurea 'Bravado' }\end{array}$ & $\begin{array}{l}0 \mathrm{~d} \\
8 \mathrm{~d}\end{array}$ & $1.1 \mathrm{~lm}$ \\
\hline $\begin{array}{l}\text { Seed minus three } \\
\text { covering layers }\end{array}$ & $\begin{array}{l}\text { E. angustifolia } \\
\text { E. purpurea 'Bravado' }\end{array}$ & $\begin{array}{l}0 \mathrm{~d} \\
0 \mathrm{~d}\end{array}$ & 1.41 \\
\hline Shoot tip & $\begin{array}{l}\text { E. angustifolia } \\
\text { E. purpurea 'Bravado' }\end{array}$ & $\begin{array}{l}100 \mathrm{a} \\
100 \mathrm{a}\end{array}$ & $0.0 \mathrm{o}$ \\
\hline Stem section & $\begin{array}{l}\text { E. angustifolia } \\
\text { E. purpurea 'Bravado' }\end{array}$ & $\begin{array}{l}38 \mathrm{c} \\
11 \mathrm{~d}\end{array}$ & 1.31 \\
\hline $\begin{array}{l}\text { ANOVA } \\
\text { Source of variation }\end{array}$ & df & \multicolumn{2}{|c|}{ Mean square } \\
\hline Species (S) & 1 & $1304.41^{* * *}$ & $0.03^{\mathrm{Ns}}$ \\
\hline $\begin{array}{l}\text { Seed covering } \\
\quad \text { removal }(\mathrm{SC}) \\
\mathrm{S} \times \mathrm{SC} \\
\text { Error }\end{array}$ & $\begin{array}{l}5 \\
5 \\
24\end{array}$ & $\begin{array}{c}8346.10^{* * * *} \\
438.54^{*} \\
3610.56\end{array}$ & $\begin{array}{l}1.73^{* * *} \\
0.12^{\text {ss }} \\
0.11\end{array}$ \\
\hline
\end{tabular}


increased in cultures of E. angustifolia and $E$. purpurea. Contamination of E. pallida was similar in cultures of intact seeds and of seeds lacking the outermost seed covering. No contamination was observed in E. angustifolia and E. pallida when two seed coverings were removed, and in all species when three were removed.

Seed germination increased from $50 \%$ to $97 \%$ as number of seed coverings removed increased from 0 to 2 (Table 1). Removal of the third layer had no additional effect.

Shoot count per explant increased from 0.5 shoots to 1.5 as the number of seed coverings removed increased from 0 to 3 .

The second analysis of data performed on data for treatments involving both seed and vegetative explants indicated that contamination was highest in cultures derived from shoottip explants, all shoot-tip cultures of $E$. angustifolia and $E$. purpurea being contaminated (Table 2). Cultures from stem explants had low contamination levels, similar to those of seed explants following removal of one, two, or three covering layers.

Shoot count was high in cultures from stem explants of both species evaluated, but did not differ significantly from those of seed explants lacking two or three seed coverings (Table 2). Cultures from shoot-tip explants produced no shoots (but all were contaminated).

Experiment 2. Cytokinin influence on shoot formation. The data for the wide range of BA concentrations indicated an interaction between BA concentration and species (Table $3)$. In general, shoot count increased with BA concentration between 0.45 and $4.45 \mu \mathrm{M}$ BA for all three species, but only in E. angustifolia did the count continue to rise between 4.45 and $44.5 \mu \mathrm{M}$ BA. Percent adventitious shoots formed increased from $0 \%$ at $0.45 \mu \mathrm{M} \mathrm{BA}$, to $83 \%$ and $100 \%$ at $4.45 \mu \mathrm{MBA}$ in E. pallida and E. angustifolia, but E. purpurea 'Bravado'

Table 3. Influence of BA concentration on shoot count, and percentage adventitious shoots on cultures derived from seed explants of three species of Echinacea.

\begin{tabular}{llcr}
\hline \hline BA $(\mu \mathrm{M})$ & \multicolumn{1}{c}{ Species } & $\begin{array}{c}\text { Shoots } \\
\text { per explant }\end{array}$ & $\begin{array}{r}\text { Adventitious } \\
\text { shoots }(\%)\end{array}$ \\
\hline 0.45 & E. angustifolia & $1.0 \mathrm{~b}^{\mathrm{z}}$ & $0 \mathrm{~b}$ \\
& E. pallida & $1.0 \mathrm{~b}$ & $0 \mathrm{~b}$ \\
4.45 & E. purpurea 'Bravado' & $3.0 \mathrm{~b}$ & $77 \mathrm{a}$ \\
& E. angustifolia & $4.0 \mathrm{ab}$ & $100 \mathrm{a}$ \\
& E. pallida & $8.0 \mathrm{a}$ & $83 \mathrm{a}$ \\
44.45 & E. purpurea 'Bravado' & $4.5 \mathrm{ab}$ & $100 \mathrm{a}$ \\
& E. angustifolia & $8.0 \mathrm{a}$ & $100 \mathrm{a}$ \\
& E. pallida & $4.5 \mathrm{ab}$ & $75 \mathrm{a}$ \\
& E. purpurea 'Bravado' & $2.5 \mathrm{~b}$ & $100 \mathrm{a}$ \\
\hline
\end{tabular}

\section{ANOVA}

Source of variation

Block

Species (S)

BA

$\mathrm{S} \times \mathrm{B}$

Error

\begin{tabular}{ccc} 
df & \multicolumn{2}{c}{ Mean square } \\
\hline 1 & $24.50^{* *}$ & $7.6^{\text {Ns }}$ \\
2 & $2.39^{\text {Ns }}$ & $2437.5^{* *}$ \\
2 & $26.06^{* *}$ & $9050.0^{* * *}$ \\
4 & $12.64^{*}$ & $1084.1^{*}$ \\
8 & 2.88 & 1809.3 \\
\hline
\end{tabular}

${ }^{2}$ Mean separation within columns by Duncan's multiple range test, $P \leq 0.05$ Ns, ${ }^{*}, * *, * * *$ Nonsignificant or significant at $P \leq 0.05,0.01$, or 0.001 , respectively.

Table 4. Influence of BA concentration on shoot count, leaf length, and root count of cultures derived from seed explants of three Echinacea species.

\begin{tabular}{|c|c|c|c|c|}
\hline $\mathrm{BA}(\mu \mathrm{M})$ & Species & $\begin{array}{c}\text { Shoots } \\
\text { per explant }\end{array}$ & $\begin{array}{c}\text { Leaf } \\
\text { length }(\mathrm{cm})\end{array}$ & $\begin{array}{c}\text { Roots } \\
\text { per explant }\end{array}$ \\
\hline$\overline{0}$ & $\begin{array}{l}\text { E. angustifolia } \\
\text { E. pallida } \\
\text { E. purpurea 'Bravado' }\end{array}$ & $\begin{array}{l}0.7 \mathrm{c}^{\mathrm{z}} \\
1.0 \mathrm{c} \\
0.9 \mathrm{c}\end{array}$ & $\begin{array}{l}2.9 \mathrm{a}-\mathrm{c} \\
3.4 \mathrm{ab} \\
1.2 \mathrm{~cd}\end{array}$ & 0.51 \\
\hline 0.09 & $\begin{array}{l}\text { E. angustifolia } \\
\text { E. pallida } \\
\text { E. purpurea 'Bravado' }\end{array}$ & $\begin{array}{l}0.8 \mathrm{c} \\
1.1 \mathrm{c} \\
1.0 \mathrm{c}\end{array}$ & $\begin{array}{l}2.9 \mathrm{a}-\mathrm{c} \\
2.7 \mathrm{a}-\mathrm{c} \\
2.4 \mathrm{a}-\mathrm{d}\end{array}$ & 0.61 \\
\hline 0.89 & $\begin{array}{l}\text { E. angustifolia } \\
\text { E. pallida } \\
\text { E. purpurea 'Bravado' }\end{array}$ & $\begin{array}{l}0.9 \mathrm{c} \\
1.1 \mathrm{c} \\
2.1 \mathrm{~b}\end{array}$ & $\begin{array}{l}0.6 \mathrm{~d} \\
3.8 \mathrm{a} \\
1.8 \mathrm{~b}-\mathrm{d}\end{array}$ & $0 \mathrm{~m}$ \\
\hline 8.89 & $\begin{array}{l}\text { E. angustifolia } \\
\text { E. pallida } \\
\text { E. purpurea 'Bravado' }\end{array}$ & $\begin{array}{l}1.2 \mathrm{c} \\
3.1 \mathrm{a} \\
1.4 \mathrm{bc}\end{array}$ & $\begin{array}{l}0.7 \mathrm{~d} \\
0.8 \mathrm{~d} \\
0.8 \mathrm{~d}\end{array}$ & $0 \mathrm{~m}$ \\
\hline
\end{tabular}

\section{ANOVA}

Source of variation

Species (S)

BA (B)

$\mathrm{S} \times \mathrm{B}$

Error

\begin{tabular}{rl} 
df & \\
\hline 2 & $5.89^{* *}$ \\
3 & $7.78^{* * *}$ \\
6 & $4.16^{* *}$ \\
29 & 1.06
\end{tabular}

Mean square

$17.00^{*}$
$26.68^{* * * *}$
$10.03^{*}$

$0.38^{\mathrm{Ns}}$

3.69

$15.47^{* * * *}$
$1.55^{\mathrm{Ns}}$

0.22

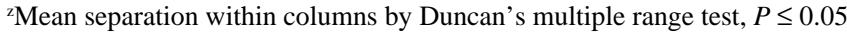

ns, ${ }^{*},{ }^{* *},{ }^{* * *}$ Nonsignificant or significant at $P \leq 0.05,0.01$, or 0.001 , respectively. produced $77 \%$ adventitious shoots at $0.45 \mu \mathrm{M}$ $\mathrm{BA}$, and $100 \%$ at 4.45 and $44.45 \mu \mathrm{M}$ BA. Adventitious shoots were not observed in subcultures following initial isolation.

Interaction was also evident in the data for the narrow range of BA concentrations (Table 4). In general, shoot count increased with BA concentration; however, shoot count was highest in E. purpurea 'Bravado' cultures at 0.89 $\mu_{\mathrm{M}} \mathrm{BA}$, but at $8.89 \mu_{\mathrm{M}} \mathrm{BA}$ in E. pallida. Data for leaf length revealed a similar interaction between BA and species (Table 4). Leaves of E. pallida were shorter at $8.89 \mu \mathrm{M} \mathrm{BA}$ than at lower concentrations, and those of $E$. angustifolia were shorter at 8.89 and $0.89 \mu \mathrm{M}$ BA than at lower concentrations, whereas leaf length in E. purpurea 'Bravado' was not significantly affected by BA concentration.

Rooting was significantly affected by BA concentration (Table 4). Cultures produced 0.5 and 0.6 roots per explant at 0 and $0.09 \mu \mathrm{M}$ BA (respectively), but none was produced at 0.89 and $8.89 \mu \mathrm{M}$ BA.

Experiment 3. Mineral composition and concentration influence on shoot formation. Shoot count per explant was higher when explants were cultured on MS (1.5 shoots) than on WPM minerals (1.2 shoots), and on half-strength ( 1.5 shoots) than on full-strength minerals (1.2 shoots) (Table 5). Leaf length was greater in E. pallida $(2.9 \mathrm{~cm})$ than in either E. angustifolia $(2.1 \mathrm{~cm})$ or E. purpurea 'Bravado' $(1.8 \mathrm{~cm})$; it also was greater on MS minerals $(2.2 \mathrm{~cm})$ than on WPM minerals $(1.8$ $\mathrm{cm})$ and on full-strength minerals $(2.6 \mathrm{~cm})$

Table 5. Main effect means and ANOVA for influence of mineral composition and concentration on shoot count and leaf length of cultures derived from seed explants of three Echinacea species.

\begin{tabular}{|c|c|c|c|}
\hline & & $\begin{array}{c}\text { Shoots } \\
\text { per explant }\end{array}$ & $\begin{array}{l}\text { Leaf } \\
\text { length } \\
(\mathrm{cm})\end{array}$ \\
\hline \multicolumn{4}{|l|}{ Species } \\
\hline \multicolumn{2}{|l|}{ E. angustifolia } & $1.34 \mathrm{a}^{\mathrm{z}}$ & $2.1 \mathrm{~b}$ \\
\hline \multicolumn{2}{|l|}{ E. pallida } & $1.23 \mathrm{a}$ & $2.9 \mathrm{a}$ \\
\hline \multicolumn{2}{|l|}{ E. purpurea } & $1.47 \mathrm{a}$ & $1.8 \mathrm{~b}$ \\
\hline \multicolumn{4}{|l|}{ Mineral source } \\
\hline \multicolumn{2}{|c|}{ Murashigue and Skoog } & 1.521 & 2.21 \\
\hline \multicolumn{2}{|c|}{ Woody Plant Medium } & $1.17 \mathrm{~m}$ & $1.8 \mathrm{~m}$ \\
\hline \multicolumn{4}{|c|}{ Mineral concentration } \\
\hline \multicolumn{2}{|l|}{ Full-strength } & $1.16 \mathrm{~s}$ & $2.6 \mathrm{r}$ \\
\hline \multicolumn{2}{|l|}{ Half-strength } & $1.51 \mathrm{r}$ & $2.0 \mathrm{~s}$ \\
\hline \multicolumn{4}{|l|}{ ANOVA } \\
\hline \multicolumn{2}{|l|}{ Source of } & \multicolumn{2}{|c|}{ Mean square } \\
\hline$\overline{\text { Block }}$ & 1 & $0.53^{\mathrm{Ns}}$ & $20.99^{*}$ \\
\hline \multicolumn{4}{|l|}{ Species (S) } \\
\hline $\begin{array}{l}\text { Mineral } \\
\quad \text { source (MS) }\end{array}$ & 1 & $7.77^{* *}$ & $56.85^{* * *}$ \\
\hline \multicolumn{3}{|l|}{ Mineral } & $20.34^{* *}$ \\
\hline $\mathrm{S} \times \mathrm{MS}$ & 2 & $4.96^{\mathrm{Ns}}$ & $21.13^{\mathrm{Ns}}$ \\
\hline $\mathrm{S} \times \mathrm{MC}$ & 2 & $0.48^{\mathrm{Ns}}$ & $11.89^{\text {Ns }}$ \\
\hline $\mathrm{MS} \times \mathrm{MC}$ & 1 & $0.87^{\mathrm{Ns}}$ & $1.00^{\mathrm{Ns}}$ \\
\hline $\mathrm{S} \times \mathrm{MS} \times \mathrm{MC}$ & 2 & $1.16^{\mathrm{Ns}}$ & $5.97^{\mathrm{Ns}}$ \\
\hline Error & 248 & 3.91 & 3.91 \\
\hline
\end{tabular}


than on half-strength minerals $(2.0 \mathrm{~cm})$.

Experiment 4. Shoot formation response to subculture frequency. Subculture frequency significantly affected total shoots per explant after 12 weeks but not shoots per subculture (Table 6). Explants subcultured at 2-week intervals produced an average of 23.9 shoots while plants subcultured at 3- and 4-week intervals produced 5.2 and 2.8 shoots, respectively.

Experiment 5. Auxin, light, and temperature influence on rooting. Rooting performance was significantly affected by species but not by light, temperature, or IBA concentration (Table 7). Root count and rooting percentage of $E$. purpurea were significantly higher than those of either E. pallida or $E$. angustifolia, and rooting percentage of $E$. angustifolia was significantly higher than that of $E$. pallida.

\section{Discussion}

This study shows that several Echinacea species can be established as aseptic shoot cultures by careful choice of explants, and subsequently multiplied by serial subculture of individual axillary shoots when adequate cytokinin is provided. Individual shoots of $E$. purpurea and E. angustifolia rooted with high and low frequency, respectively, while $E$. pallida did not respond to the rooting treatments used in this study.

Table 6. Influence of subculture frequency on shoots per subculture and total shoots produced after 12 weeks of serial subculturing of E. angustifolia embryo explants.

\begin{tabular}{lcr}
\hline \hline $\begin{array}{l}\text { Subculture } \\
\text { frequency (wk) }\end{array}$ & $\begin{array}{l}\text { Shoots per } \\
\text { subculture }\end{array}$ & $\begin{array}{r}\text { Total } \\
\text { shoots }\end{array}$ \\
\hline 2 & $1.8 \mathrm{a}^{\mathrm{z}}$ & $23.9 \mathrm{a}$ \\
3 & $1.6 \mathrm{a}$ & $5.2 \mathrm{~b}$ \\
4 & $1.4 \mathrm{a}$ & $2.8 \mathrm{~b}$ \\
\hline zMean separation within columns by Duncan's mul
\end{tabular}

Mean separation within columns by Duncan's multiple range test, $P \leq 0.05$

Table 7. Influence of species, light, temperature, and IBA concentration on rooting percentage and root count of shoots from seed explants after long-term serial subculture of three Echinacea species.

\begin{tabular}{lcc}
\hline \hline Species & $\begin{array}{c}\text { Rooting } \\
(\%)\end{array}$ & $\begin{array}{c}\text { Roots } \\
\text { per explant }\end{array}$ \\
\hline E. angustifolia & $32 \mathrm{~b}^{\mathrm{z}}$ & $0.8 \mathrm{~b}$ \\
E. pallida & $3 \mathrm{c}$ & $0.0 \mathrm{~b}$ \\
E. purpurea 'Bravado' & $95 \mathrm{a}$ & $6.8 \mathrm{a}$ \\
\hline
\end{tabular}

ANOVA

Source

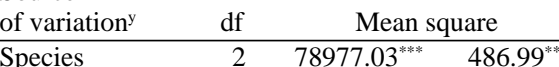

$\begin{array}{lccc}\text { Species } & 2 & 78977.03^{* * *} & 486.99 \\ \text { Light } & 1 & 155.11^{\text {Ns }} & 65.60^{\text {Ns }}\end{array}$

$\begin{array}{llll}\text { Temp } & 1 & 356.54^{\mathrm{Ns}} & 96.69^{\mathrm{Ns}}\end{array}$

$\begin{array}{llll}\text { IBA } & 2 & 66.73^{\mathrm{Ns}} & 96.94^{\mathrm{Ns}}\end{array}$

Error 435.73

88.66

${ }^{2}$ Mean separation within columns by Duncan's multiple range test, $P \leq 0.05$

y No interaction effects were significant at $P \leq 0.05$. ns, **,*** Nonsignificant or significant at $P \leq 0.01$ or 0.001 , respectively.
Success of aseptic culture isolation was dependent on the initial explant used. When treated with a $15 \%$ commercial bleach solution, shoot tips were most prone to contamination, stem segments less prone, and excised embryos essentially contaminant-free. These observations suggest that the contaminating agents were surface, rather then systemic, in origin. Therefore, isolation of stock plant material in extremely clean conditions should reduce the contamination resulting from these explant types. Species differed with regard to frequency of contamination of seed explants, but this may be related to variability among seed sources, storage conditions, or collection sites. Removal of two of the three seed covering layers eliminated almost all contamination.

One benefit of removing seed coverings from embryos is the elimination of seed dormancy. Dormancy, which can be overcome by stratification, has been described for Echinacea species (Baskin et al., 1992; Bratcher et al., 1993; Feghahati and Reese, 1994; Wartidiningsih and Geneve, 1994; Wartidiningsih et al., 1994). Mean germination of all intact seeds used in this study was low $(50 \%)$ and that of individual species was as low as $28 \%$ (E. angustifolia). The site of inhibition seemed to be in the outer two layers. Removal of these resulted in a mean germination of $97 \%$ for the three species combined. Inhibition of germination in intact seeds was not solely a function of contamination. We have observed in ongoing experiments that germination rates of intact seed on watersoaked blotter paper were low, even though contamination did not occur. However, germination of excised embryos of the same species on the same blotter was nearly $100 \%$ (data not shown). Feghahati and Reese (1994) showed that the seed stratification requirement for germination of intact Echinacea seed could be eliminated by treatment with ethylene. Taken together, these findings suggest that nonmechanical germination inhibitors involved in seed dormancy of some Echinacea species reside in the outer two seed covering layers.

Cytokinin concentration is critical during shoot-culture establishment from seed explants because even moderate levels of BA $(4.45 \mu \mathrm{M})$ induced a large number of adventitious shoots. This may not be desirable for routine micropropagation because of the greater tendency of adventitious shoots to undergo somatic mutation. The observation that adventitious shoots were not detected in subcultures following initial isolation of seed explants suggests that embryos are more likely to form adventitious shoots in response to cytokinin than are shoots formed after establishment. Reducing BA level to $0.89 \mu \mathrm{M}$ during establishment eliminated adventitious shoot formation in two of the three species examined, but eliminated axillary shoot multiplication as well. Serial subculturing at this cytokinin level induces axillary shoot multiplication. When E. angustifolia was subcultured at $0.89 \mu \mathrm{M}$ BA, a 2-week subculture frequency resulted in a multiplication rate of 1.8 shoots per culture. Higher concentrations of cytokinin may be possible during subculture, as the tendency to form adventitious shoots seems to be less after the initial explant isolation cycle.

Mineral composition and content were important for optimizing culture performance. Higher shoot production and greater leaf size were obtained on medium with MS minerals than on one with WPM minerals. Using a halfstrength medium improved shoot production at the expense of leaf size; this benefit disappeared after species had been subcultured three or four times, while leaf size remained higher in full-strength medium. Therefore, fullstrength medium should be used for postisolation subculture.

Induction of root formation differed among species. Echinacea purpurea, a species with a fibrous root system when grown in the field, was easily rooted by eliminating cytokinin from the medium. Auxin was not necessary for root induction and did not significantly affect the number of roots produced. Rooting response of $E$. angustifolia was poor and $E$. pallida produced no roots. Both of these species produce taproot systems in the field and may require different root induction signals than do fibrous-rooted species. More work on root induction is required to optimize micropropagation of these two species.

Symptoms of aster yellows disease have long been observed in ornamental and medicinal plantings of Echinacea. Recently, the causal agent for this disease, a phytoplasma, was positively identified in phloem cells of Echinacea angustifolia and E. purpurea (Hwang et al., 1997; Khadhair et al., 1997). Incidence of the disease was much lower in $E$. angustifolia, suggesting natural resistance. Clonal propagation using the micropropagation methods developed in this study can provide a means of rapid multiplication of improved selections resistant to this disease and for maintaining disease-free germplasm.

\section{Literature Cited}

Baskin, C.C., J.M. Baskin, and G.R. Hoffman. 1992. Seed dormancy in the prairie forb. Echinacea angustifolia var. angustifolia (Asteraceae): Afterripening pattern during cold stratification. Intl. J. Plant Sci. 153:239-243.

Bratcher, C.B., J.M. Dole, and J.C. Cole. 1993. Stratification improves seed germination of five native wildflower species. HortScience 28:899_ 901

Bridgen, M. 1986. Do-it-yourself cloning. Greenhouse Grower 4:43,46-47.

Feghahati, J.S.M., and R.N. Reese. 1994. Ethylene, light-, and prechill-enhanced germination of Echinacea angustifolia seeds. J. Amer. Soc. Hort. Sci. 119:853-858.

Foster, S. 1985. Echinaceas: The purple coneflowers. Amer. Hort. 64:14-17.

Foster, S. 1990. Echinacea-Beauty and medicine for your garden. Herb Companion, Oct./Nov., p. 33-38.

Hwang, S.F., K.F. Chang, R.J. Howard, A.H. Khadhair, R.G. Gaudiel, and C. Hiruki. 1997. First report of a yellows phytoplasma disease in purple coneflower (Echinacea spp.) Can. J. Plant Dis. Prot. 104:182-192.

Khadhair, A.H., S.F. Hwang, K.F. Chang, and R.J. Howard. 1997. Molecular identification of aster 
yellows phytoplasma in purple coneflower and monarda based on PCR amplification and RFLP analyses of $16 \mathrm{~S}$ rDNA sequences. J. Plant Dis. Prot. 104:403-410.

Kindscher, K. 1989. Ethnobotany of purple coneflower (Echinacea angustifolia, Asteraceae) and other Echinacea species. Econ. Bot. 43:498507.

Lineberger, D. 1983. Micropropagation for the perennial industry, p. 7-9. In: E.M. Smith and S.M. Still (eds.). Proc. Herbaceous Perennial Symp., Columbus, Ohio. Ohio Coop. Ext. Ser. Bul. 717.

Lloyd, G. and B. McCown. 1980. Commercially feasible micropropagation of mountain laurel,
Kalmia latifolia, by use of shoot-tip culture. Comb. Proc. Intl. Plant Prop. Soc. 30:421-427. McGregor, R.L. 1968. Taxonomy of the genus Echinacea (Compositae). Univ. of Kansas Sci. Bul. 48:113-142.

Murashige, T. and F. Skoog. 1962. A revised medium for rapid growth and bioassays with tobacco tissue cultures. Physiol. Plant. 15:473497.

Rawls, R. 1996. Europe's strong herbal brew. Chem. Eng. News, 23 Sept., p. 53-60.

Rhodus, T. 1995. Top 20 perennials. Greenhouse Grower. Jan. p. 80-84.

Shemluck, M. 1982. Medicinal and other uses of the
Compositae by Indians in the United States and Canada. J. Ethnopharm. 5:303-358.

Starman, T.W., T.A. Cerny, and A.J. MacKenzie. 1995. Productivity and profitability of some field-grown specialty cut flowers. HortScience 30:1217-1220.

Wartidiningsih, N. and R.L. Geneve. 1994. Seed source and quality influence germination in purple coneflower [Echinacea purpurea (L.) Moench.]. HortScience 29:1443-1444.

Wartidiningsih, N., R.L. Geneve, and S.T. Kester. 1994. Osmotic priming or chilling stratification improves seed germination of purple coneflower. HortScience 29:1445-1448. 